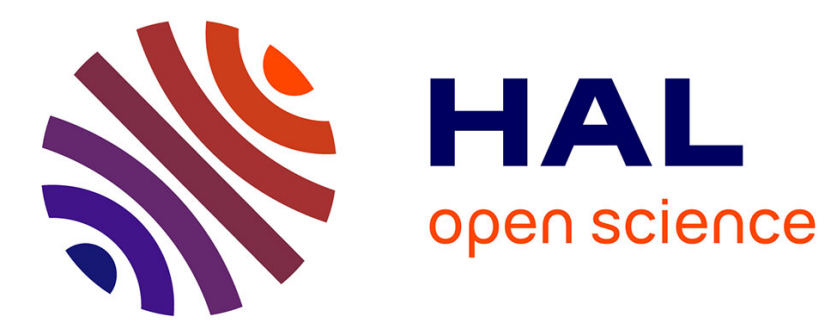

\title{
Joint diffusivity and source estimation in tokamak plasma heat transport
}

Sarah Mechhoud, Emmanuel Witrant, Luc Dugard, Didier Moreau

\section{To cite this version:}

Sarah Mechhoud, Emmanuel Witrant, Luc Dugard, Didier Moreau. Joint diffusivity and source estimation in tokamak plasma heat transport. ACC 2013 - American Control Conference, Jun 2013, Washington, DC, United States. pp.0335. hal-00931657

\section{HAL Id: hal-00931657 https://hal.science/hal-00931657}

Submitted on 15 Jan 2014

HAL is a multi-disciplinary open access archive for the deposit and dissemination of scientific research documents, whether they are published or not. The documents may come from teaching and research institutions in France or abroad, or from public or private research centers.
L'archive ouverte pluridisciplinaire HAL, est destinée au dépôt et à la diffusion de documents scientifiques de niveau recherche, publiés ou non, émanant des établissements d'enseignement et de recherche français ou étrangers, des laboratoires publics ou privés. 


\title{
Joint diffusivity and source estimation in tokamak plasma heat transport*
}

\author{
Sarah Mechhoud ${ }^{1}$, Emmanuel Witrant $^{1}$, Luc Dugard $^{1}$ and Didier Moreau ${ }^{2}$
}

\begin{abstract}
In this work, we focus on the diffusivity and source identification in the electron heat transport model. This phenomenon is described by a second-order parabolic differential equation with distributed diffusion parameter and input. Once existence and uniqueness conditions of the heat model solution are established, a spectral Galerkin method is used to express this solution in the finite dimensional framework. The time-space separation and the Kalman filter are combined to simultaneously estimate the distributed variables (diffusion coefficient and the input). Computer simulations on both simulated and real data are provided to illustrate the performance of the proposed technique.
\end{abstract}

\section{INTRODUCTION}

Distributed parameter systems (DPS) widely exist in industrial processes. These physical and chemical systems are governed by partial differential equations (PDE) and complex spatio-temporal nonlinear dynamics. In many situations, it is difficult to get an accurate nominal PDE description due to incomplete physical or chemical knowledge (unknown system parameters, unknown disturbances...). These uncertainties make the modelling problem tedious. A tokamak is complex physical system composed of several DPS processes. In this work, we consider heat transport. It is one of the main issues in controlled fusion research. The heat transport is described by a one-dimensional diffusion equation in a cylinder, where electrons and ions heat diffusivity are distributed (time and space-varying). Several empirical models for the diffusion coefficient in hot plasmas exist. They depend on several conditions (tokamak dimensions, discharge parameters and temperature profile to name a few), but all of them assert the diffusivity dependence on temperature gradient, magnetic and velocity shears. As a consequence, the heat model becomes nonlinear, complex and coupled with other variables. Another unknown quantity is the heating energy absorbed by the particles or what we call the source term. It depends on the power deposition profiles and efficiencies of the various heating systems (radiofrequency waves and high-energy neutral beams), and is sometimes difficult to model because of parasitic phenomena and anomalous energy losses. To derive an experimentallybased model, the diffusion coefficient is assumed to be an

This project receives partial funding provided by the Bonus Quality Research of Grenoble-INP.

${ }^{1}$ S. Mechhoud, E. Witrant and L. Dugard are with UJFGrenoble 1/CNRS, Grenoble Image Parole Signal Automatique (GIPSA-lab), UMR 5216, B.P. 46, F-38402 St Martin d'Hères, France \{sarah.mechhoud, emmanuel.witrant, luc.dugard @gipsa-lab.grenoble-inp.fr

${ }^{2}$ D. Moreau is with CEA, IRFM, F-13108 Saint Paul-Lez-Durance, France didier.moreau@cea.fr unknown variable and we aim to reconstruct this coefficient and the source term using parameter identification tools. These parameters are very important for the development of current and pressure profile control strategies. In general, estimating a partial differential equation (PDE) in an infinite dimensional framework is a challenging task. We thus first convert the PDE into a finite dimensional system. This system can be derived using various numerical methods like finite difference method (FDM), finite element methods (FEM), spectral methods (SM) and others. We consider the spectral method in the Galerkin formulation scheme. It uses a set of global expansion functions for the approximate solution. Thus few parameters are needed to represent a smooth approximate solution. Since the diffusion parameter and the source term are space-time variables, each one is projected on its appropriate finite dimensional subspace. Hence, the PDE is converted into a set of ordinary differential equations (ODE), which can be temporally discretized in order to obtain a discrete-time system and to be able to choose an appropriate identification method. Aiming to estimate states, parameters and the source term, the problem becomes nonlinear. One of the most well-known and trustworthy nonlinear filters is the extended Kalman filter (EKF), based on the linearization of the nonlinear dynamics around the posterior/prior estimated state with the first order Taylor series expansion. In the standard EKF, the deterministic inputs in the model should be known. Otherwise, a high bias is introduced into the state estimation. In [1], [2], [3], the approach was to use a minimum-variance-unbiased (MVU) estimator to reconstruct both states and unknown inputs and only the MVU optimality was proven. In [4], a novel approach that considers the unknown inputs as a part of the state instead of disturbances was presented. By minimising the weighted least squares objective function with respect to an extended variable including states and unknown inputs, the proposed filter is a more general extension of the EKF. It guarantees, under the observability condition, the global optimality for both state and unknown inputs estimation in a least square sense. When there is no direct relationship between the inputs and the outputs, the filter was named by its authors the Extended Kalman Filter with Unknown Inputs Without Direct Feed through (EKF-UI-WDF). For a nonlinear problem, the EKF-UI-WDF is still a first order linearization of the dynamics. While other filters like the Unscented Kalman filter can approximate the nonlinearities at least to the second order, the simplicity of the EKF's implementation (compared to the UKF) motivated us to select it as a first approach. 
This paper is organized as follows: Section II describes the heat transport model in plasma, the existence and uniqueness of the problem solution and the continuous spectral Galerkin formulation. The identification problem formulation and the EKF-UI-WDF application are presented in Section III. Both simulations with computed and real measurements data are provided to illustrate the performance of the chosen approach. The conclusion and future work are presented in Section IV.

\section{ELECTRON HEAT TRANSPORT MODEL}

Assuming the poloidal and toroidal axisymmetry, the tokamak is considered as an infinite cylinder where space variations occur only along the radius $\mathrm{r}(r \leq a)$. Applying the energy conservation principle, neglecting the spatiotemporal variations of the electron density with respect to the temperature variations and using the normalized variable $z=\frac{r}{a}$, the electron heat transport model is given by the following parabolic partial differential equation [5]:

$$
\left\{\begin{array}{l}
\frac{\partial T}{\partial t}=\frac{1}{z} \frac{\partial}{\partial z}\left(z \chi_{e}(z, t) \frac{\partial T}{\partial z}\right)-\frac{1}{\tau} T(z, t)+S(z, t) \\
\frac{\partial T}{\partial z}(z=0, t)=0 ; T(z=1, t)=0 \\
\left.T(z, t=0)=0 ; \quad z \in \Omega ; \quad t \in] 0, t_{f}\right]
\end{array}\right.
$$

where $t$ is the time, $\Omega$ is the interval $] 0,1\left[, \chi_{e}\right.$ is the electron diffusion, $\tau(<\infty)$ is a damping time modelling the energy losses, $T$ is the electron temperature and $S$ is the power density absorbed by the particles from an external heating system.

In system (1), the second and third equations represent initial and boundary conditions, chosen to guaranty the symmetry and boundedness of the solution near zero.

\section{A. Existence and uniqueness of the solution}

First, let us introduce these Hilbert spaces:

$L^{2}(\Omega)=\left\{f: \int_{\Omega} f^{2} \partial \Omega<\infty\right\}$ and its usual norm $\|\cdot\|_{0}$,

$$
H_{0,\{1\}}^{1}(\Omega)=\left\{f \in L^{2}(\Omega):\left.f\right|_{1}=0, \nabla f \in L^{2}(\Omega)\right\}
$$

endowed with the inner product $(.,$.$) and the norm \|.\|_{1}$, defined respectively as follows:

$$
\left\{\begin{array}{l}
\forall f, g \in\left(H_{0,\{1\}}^{1}(\Omega)\right)^{2}:(f, g)=\int_{\Omega} f g \partial \Omega . \\
\forall f \in H_{0,\{1\}}^{1}(\Omega):\|f\|_{1}=\|f\|_{0}+\|\nabla f\|_{0} .
\end{array}\right.
$$

Let $X e$ be a set defined as $\forall x \in \Omega, \forall t \in\left[0, t_{f}\right]$ :

$$
X e=\left\{f \in L^{2}\left(0, t_{f} ; L^{2}(\Omega)\right): f(x, t)>c>0\right\}
$$

To get the variational formulation of this problem, we first multiply equation (1) by a test function $v(z) \in H_{0,\{1\}}^{1}(\Omega)$ and then integrate on $\Omega$. Using the Green formula and the boundary conditions leads to:

$$
\begin{aligned}
\int_{0}^{1} \frac{\partial T}{\partial t} v(z) d z=- & \int_{0}^{1} \chi_{e}(z, t) \frac{\partial T}{\partial z} \frac{d v}{d z} d z-\frac{1}{\tau} \int_{0}^{1} T(z, t) v(z) d z \\
& +\int_{0}^{1} S(z, t) v(z) d z
\end{aligned}
$$

Let us associate with $T$ the mapping $\mathbf{T}(t)$ :

$$
\mathbf{T}:\left[0, t_{f}\right] \quad \longrightarrow \quad H_{0,\{1\}}^{1}(\Omega)
$$

defined by: $\mathbf{T}(t):=T(z, t)$

The bilinear form $b$ on $H_{0,\{1\}}^{1}(\Omega)$ is given by:

$$
\begin{aligned}
b: H_{0,\{1\}}^{1}(\Omega) \times H_{0,\{1\}}^{1}(\Omega) & \longrightarrow \mathbb{R} \\
(\mathbf{T}, v) \longmapsto & \int_{0}^{1} \chi_{e}(z, t) \frac{\partial T}{\partial z} \frac{d v}{d z} d z \\
& +\frac{1}{\tau} \int_{0}^{1} T(z, t) v(z) d z
\end{aligned}
$$

and $L$ is a linear form defined on $L^{2}\left(0, t_{f} ; H^{-1}(\Omega)\right)$ such that:

$$
\forall v \in H_{0,\{1\}}^{1}(\Omega), L(t)(v)=\int_{0}^{1} S(z, t) v(z) d z
$$

where $H^{-1}(\Omega)$ is the dual space of $H_{0,\{1\}}^{1}(\Omega)$.

Equation (3) can then be written as:

$\left\{\begin{array}{l}\frac{d}{d t}(\mathbf{T}(t), v)-b(\mathbf{T}(t), v)=L(t)(v) ; \quad \forall v \in H_{0,\{1\}}^{1}(\Omega) \\ \mathbf{T}(0)=T(x, 0)=0\end{array}\right.$

and it is the weak formulation of problem (1).

Theorem1: Under the previous definitions and for all $\chi_{e}$ in $X e, \tau$ in $\mathbb{R}_{+}^{*}$ and $S$ in $L^{2}\left(0, t_{f} ; L^{2}(\Omega)\right)$, the system (4) admits a unique solution in $L^{2}\left(0, t_{f} ; H_{0,\{1\}}^{1}(\Omega)\right)$. This solution is stable with respect to the data $\left(\chi_{e}, \tau, S\right)$.

Proof: First note that for the bilinear form $b$ :

$\forall \chi_{e} \in X e, \tau \in \mathbb{R}_{+}^{*}:$

$$
|b(T, v)| \leq\left(\left\|\chi_{e}(x, t)\right\|_{0}+\frac{1}{\tau}\right)\|v\|_{1}\|T\|_{1}
$$

from the Cauchy-Schwartz inequality and norm's $\|.\|_{1}$ definition, $|$.$| is the absolute value operator. Second:$

$$
\forall v \in H_{0,\{1\}}^{1}(\Omega), \forall \chi_{e} \in X e:|b(v, v)| \geq \frac{c}{\sqrt{(1+C(\Omega))}}\|v\|_{1}
$$

where $C(\Omega)$ is the Poincaré constant. Hence, the bilinear form $b$ is continuous and $H_{0,\{1\}}^{1}$-coercive.

For the linear form $L$ :

$$
\forall S \in L^{2}\left(0, t_{f} ; L^{2}(\Omega)\right):|L(t)(v)| \leq\|S(x, t)\|_{0}\|v(x)\|_{1}
$$

then, $L$ is continuous and using the Lax-Milgram theorem, the results in Theoreml are guaranteed. This concludes the proof.

To get the classical solution of Eq.(1): $T(z, t) \in$ $C^{1}\left(0, t_{f} ; C^{2}(\Omega)\right)$, the following regularity conditions have to be fulfilled:

$$
\left\{\begin{array}{l}
S \in C^{0}\left(0, t_{f} ; C^{0}(\Omega)\right) \\
\chi_{e} \in C^{0}\left(0, t_{f} ; C^{1}(\Omega)\right)
\end{array}\right.
$$

(3Note that these regularity conditions are not restrictive and are consistent with the physical properties of $\chi_{e}$ and $S$. 


\section{B. Spectral Galerkin formulation}

Once the well-posedness of the heat model is asserted, an approximate solution can be derived.

As mentioned in the introduction, we are working in the Galerkin formulation framework, where the residuals of the approximation are made orthogonal to the space of the approximate solution. An efficient method that leads to a low-order model is the spectral-Galerkin method. In this technique, it is assumed that the exact solution of system (1) can be expressed as an infinite sum of global shape functions $\left\{w_{i}(x)\right\}_{i=0}^{\infty}$ multiplied by their corresponding weighting coefficients $\left\{x_{i}(t)\right\}_{i=0}^{\infty}$. These shape functions are generally chosen to form an orthonormal basis of the solution space. Then, the approximate solution is extracted by truncating this expression.

Consequently, for a fixed $n$, the finite dimensional space of approximation $V_{n}$ is given by:

$$
\left\{\begin{array}{l}
V_{n}=\operatorname{span}\left\{\omega_{1}, \omega_{2}, \ldots, \omega_{n}\right\} \\
V_{n} \subset V_{n+1} \text { et } \overline{\cup V_{n}}=H_{0,\{1\}}^{1}(\Omega)
\end{array}\right.
$$

and the restriction of $T(z, t)$ and $v(z)$ on $V_{n}$ gives:

$$
\left\{\begin{array}{l}
T(z, t)=\sum_{k=0}^{n} x_{k}(t) \omega_{k}(z) \\
v(z)=\sum_{k=1}^{n} \alpha_{k} \omega_{k}(z)
\end{array}\right.
$$

By substituting (5) in (4), the PDE (1) is converted into a set of ODEs in the continuous time domain given by:

$$
\left\{\begin{array}{l}
\dot{X}(t)=A(t) X(t)+B(t) \\
X(0)=0
\end{array}\right.
$$

where: $X(t)=\left[x_{1}(t), x_{2}(t), \ldots, x_{n}(t)\right]^{T}\left(X \in \mathbb{R}^{n}\right)$, $A(t) \in \mathbb{R}^{n \times n}$ is the matrix of dynamics, symmetric and given by:

$A(t)=-\left(\begin{array}{ccc}\int_{0}^{1} \chi_{e}(z, t) \omega_{1}^{\prime 2}(z) d z+\frac{1}{\tau} & \ldots & \int_{0}^{1} \chi_{e}(z, t) \omega_{1}^{\prime}(z) \omega_{n}^{\prime}(z) d z \\ \vdots & \ddots & \vdots \\ \int_{0}^{1} \chi_{e}(z, t) \omega_{1}^{\prime}(z) \omega_{n}^{\prime}(z) d z & \ldots & \int_{0}^{1} \chi_{e}(z, t) \omega_{n}^{\prime 2}(z) d z+\frac{1}{\tau}\end{array}\right)^{\operatorname{la}}$

and the input vector $B(t) \in \mathbb{R}^{n}$ is given by:

$$
B(t)=\left(\begin{array}{c}
\int_{0}^{1} S(z, t) \omega_{1}(z) d z \\
\vdots \\
\int_{0}^{1} S(z, t) \omega_{n}(z) d z
\end{array}\right)
$$

The basis functions $w_{k}(x)$ should be carefully selected. The most popular ones are the Fourier series, the orthogonal polynomials and the eigenfunctions of the PDE operator.

Since the Fourier series are suited for periodic problems (otherwise the exponential convergence is no longer guaranteed) and the orthogonal polynomials lead to a set of algebraic differential equations (these polynomials do not naturally satisfy the boundary conditions), we chose the eigenfunctions of the Laplace-Dirichlet-Neumann operator as the basis functions of our problem. These eigenfunctions can be seen as some modified Fourier series [6] and form an orthonormal basis of $H_{0,\{1\}}^{1}$. They are given by:

$$
w_{k}(x)=\cos \left(\frac{\pi}{2} x(2 k-1)\right), \quad k=1,2, \ldots, n .
$$

As our partial differential operator is spatio-temporal, the generalization of the results on modified Fourier series for aperiodic systems found in [6] is not straightforward. Numerical simulations show that the expansion coefficients decay like $\mathcal{O}\left(n^{-1}\right)$.

To measure the error due to the approximation technique, define the projection relative error (PRE) as:

$$
\left\{\begin{array}{l}
\operatorname{PRE}(z, t)=\frac{T_{e x p}(z, t)-T_{\text {rec }}(z, t)}{T_{\exp p}(z, t)}, \quad z \in[0,0.8] \\
T_{r e c}(z, t)=\sum_{i=1}^{n}\left(\int_{0}^{1} T_{\exp }(z, t) w_{i}(z) d z\right) w_{i}(z)
\end{array}\right.
$$

where: $T_{r e c}$ is the temperature profile reconstructed after projection of the measured temperature $T_{\exp }$ on the space $V_{n}$. As the electron temperature model (1) does not include the edge pedestal and plasma scrape-off phenomena, the PRE is defined for $z$ belonging to the interval $[0,0.8]$. Fig. 1

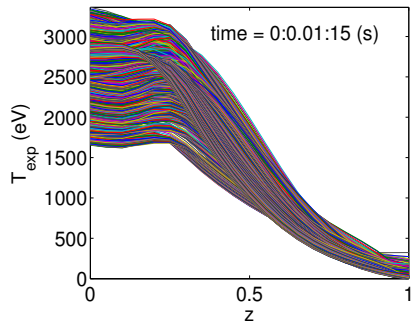

(a)

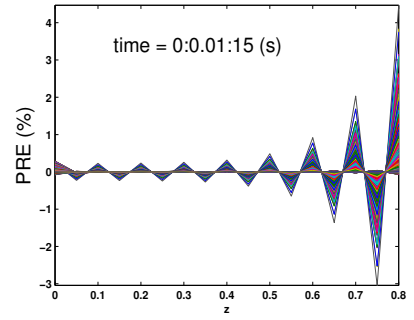

(b)
Fig. 1: (a) Spatio-temporal temperature $T_{\exp }$ and its PRE (b).

illustrates the PRE using the Tore Supra experimental data TS 33632 with $n=20$. The closer we get to the edge, the larger is the PRE, but it is still below the $5 \%$ tolerated level.

\section{JOINT DIFFUSION AND SOURCE ESTIMATION}

In this section, we focus on estimating simultaneously the diffusion coefficient and the source term ( $\chi_{e}$ and $S$, respectively). The fundamental identification problem consists in finding $\chi_{e} \in X e, S \in L^{2}\left(0, t_{f} ; L^{2}(\Omega)\right)$ which minimize the following distributed least-squares criterion:

$J\left(\chi_{e}, S\right)=\int_{0}^{t_{f}} \int_{0}^{1}\left(T_{e x p}(z, t)-T\left(z, t ; \chi_{e} ; S\right)\right)^{2} d z d t$

where $T_{\exp }(z, t)$ is the experimental temperature profile and $T\left(z, t ; \chi_{e} ; S\right)$ is the solution of (1). It has been proved in [7] that, since the classical solution depends continuously on the parameters $\left(\chi_{e}\right.$ and $S$ ) due to the operator coercivity, the approximate solution of (6) converges to the original one of (1). Using the Ritz formulation, the estimation problem (7) can be formulated as an approximate parameter estimation problem given by:

$$
J^{n}\left(\chi_{e_{M}}, S_{L}\right)=\int_{0}^{t_{f}}\left(y_{\exp }(t)-y\left(t ; \chi_{e_{M}} ; S_{L}\right)\right)^{2} d t
$$


where $y\left(t ; \chi_{e_{M}} ; S_{L}\right)$ is the output of the state space system (6) and $y_{\text {exp }}$ is given by:

$$
\left\{\begin{array}{l}
y_{\text {exp }}(t)=\left[y_{1_{\text {exp }}}(t), \ldots, y_{n_{\text {exp }}}(t)\right]^{T} \\
y_{k_{\text {exp }}}(t)=\int_{0}^{1} T_{\text {exp }}(z, t) \omega_{k}(z) d z, \quad k=1, \ldots, n
\end{array}\right.
$$

The vector $y_{\exp }$ forms the weighting coefficients coming from the projection of the experimental profile data. $\left\{\chi_{e_{M}}\right\}$ and $\left\{S_{L}\right\}$ are parameters sequences that converge to the parameters solution $\left(\chi_{e}, S\right)$ under the compactness condition on the chosen parameters set (see section III in [7] for the proof).

Since there exists sequences of orthonormal polynomials in $L^{2}(\Omega)$, for example the well-known Legendre polynomials, the diffusion parameter $\chi_{e_{M}}(z, t)$ can be expressed as follows:

$$
\chi_{e_{M}}(z, t)=\sum_{k=0}^{M} \alpha_{k}(t) p_{k}(z)=\theta^{T}(t) P(z) .
$$

where $\left\{p_{i}(z)\right\}_{i=0}^{M}$ can be seen as global shape functions, and:

$$
\left\{\begin{array}{l}
\theta^{T}(t)=\left[\alpha_{1}(t), \alpha_{2}(t), \ldots, \alpha_{M}(t)\right] \quad, \quad \theta \in \mathbb{R}^{M} \\
P(z)=\left[p_{1}(z), p_{2}(z), \ldots, p_{M}(z)\right]^{T}
\end{array}\right.
$$

The power deposition profiles that form the source term in the heat diffusion equation can often be approximated using a Gaussian distribution, and it is difficult to represent it accurately using orthogonal modal functions. We thus select the cubic B-splines functions as an expansion basis:

$$
S_{L}(z, t)=\sum_{k=0}^{L} \zeta_{k}(t) b_{k}(z)=\mathcal{B}(z) \beta(t)
$$

where :

$$
\left\{\begin{array}{l}
\beta(t)=\left[\zeta_{1}(t), \zeta_{2}(t), \ldots, \zeta_{L}(t)\right]^{T} \quad, \quad \beta \in \mathbb{R}^{L} \\
\mathcal{B}(z)=\left[b_{1}(z), b_{2}(z), \ldots, b_{L}(z)\right]
\end{array}\right.
$$

The matrix $A(t)$ defined in Section II-B then becomes:

$$
A\left(\theta^{T}(t)\right)=-\theta^{T}(t) \otimes\left(\begin{array}{c}
\int_{0}^{1} P(z) \omega_{1}^{\prime 2}(z) d z+\frac{1}{\tau} \cdots \int_{0}^{1} P(z) \omega_{1}^{\prime}(z) \omega_{n}^{\prime}(z) d z \\
\vdots \\
\ddots \\
\int_{0}^{1} P(z) \omega_{1}^{\prime}(z) \omega_{n}^{\prime}(z) d z \cdots \int_{0}^{1} P(z) \omega_{n}^{\prime 2}(z) d z+\frac{1}{\tau}
\end{array}\right)
$$

And (6) can be written as a linear parameter-varying system:

$$
\left\{\begin{array}{l}
\dot{X}(t)=A\left(\theta^{T}(t)\right) X(t)+\mathcal{D} \beta(t), \quad X(0)=0 \\
y(t)=X(t)
\end{array}\right.
$$

where $\mathcal{D} \in \mathbb{R}^{n \times L}$ has the following form:

$$
\mathcal{D}=\left(\begin{array}{ccc}
\int_{0}^{1} \omega_{1}(z) b_{1}(z) d z & \cdots & \int_{0}^{1} \omega_{1}(z) b_{L}(z) d z \\
\vdots & \ddots & \vdots \\
\int_{0}^{1} \omega_{n}(z) b_{1}(z) d z & \cdots & \int_{0}^{1} \omega_{n}(z) b_{L}(z) d z
\end{array}\right)
$$

This approach is different from the one presented in [7], where the authors were interested only in parameters estimation and where the projection operation was done simultaneously in time and space. This leads to a purely nonlinear parameter estimation problem. The main advantage of our formulation is to deal with only time-varying coefficients. Note that, in previous works [8],[9], where the problem was attempted to be solved in the finite dimensional framework, the source term was considered as a known variable.

\section{Remark:}

The initial problem which consisted in estimating distributed parameters in an infinite dimensional system is converted, thanks to the Ritz-Galerkin formulation, to a finite dimensional estimation problem. Thus, process identification techniques developed for state space systems can be used to estimate both $\theta$ and $\beta$ of system (12). The Kalman filter is one of the widely used estimation techniques to jointly estimate inputs and parameters.

\section{A. Estimation using the EKF-UI-WDF}

When the system inputs are not known, the KF-UI-WDF provides an efficient observer to reconstruct them. The recursive solution of this filter for estimating the states and the inputs was first derived and presented in [4]. The problem amounts to first minimize a weighted lest-squares objective function (without any constraint or a priori knowledge of the inputs) with respect to the actual states and all the inputs from the initial time to the actual instant and then find a recursive way to do this sequentially without consuming storage memory.

In this section the KF-UI-WDF is extended to estimate also the parameters using the same philosophy as the EKF. To this end, we first extend the state vector in (12) to include the unknown parameter $\theta$ and then discretize the time-variation. Let:

$f(X(t), \theta(t))=\left(\begin{array}{c}\left(I+d t * A\left(\theta^{T}(k)\right)\right) X(k)+d t * \mathcal{D} \beta(k) \\ \theta(k)\end{array}\right)$

The discrete extended model is given by:

$\left\{\begin{array}{l}x_{e x t}(k+1)=f(X(t), \theta(t))+\left(\begin{array}{c}w(k) \\ \\ 0\end{array}\right) \\ y(k)=\left[\begin{array}{ll}I_{n} & 0\end{array}\right] x_{\text {ext }}(k)+v(k)=C x_{\text {ext }}(k)+v(k) .\end{array}\right.$

where: $x_{\text {ext }}(k+1)=\left(\begin{array}{c}X(k+1) \\ \theta(k+1)\end{array}\right)$ is the extended state, $C=$ $\left[\begin{array}{ll}I_{n} & 0\end{array}\right]$ is the observation matrix, $d t$ is the time step, $w(k) \in$ $\mathbb{R}^{n}$ and $v(k) \in \mathbb{R}^{n}$ are respectively the model uncertainty and the measurements noise vectors, assumed to be independent, white and Gaussian. They are characterized by:

$$
\begin{gathered}
\mathbb{E}(w)=\mathbb{E}(v)=0, \mathbb{E}\left(w(i) w^{T}(j)\right)=W \delta_{i j}, \\
\mathbb{E}\left(v(i) v^{T}(j)\right)=V \delta_{i j}, \mathbb{E}\left(v(i) w^{T}(j)\right)=0 .
\end{gathered}
$$

Based on the above representation (13)-(14), the EKF-UIWDF approach can be used to estimate the extended state $\hat{x}_{e x t}(k \mid k)$ and the inputs $\hat{\beta}(k-1 \mid k)$ given all the observations taken up to time $k$. The EKF asymptotic convergence behaviour for observable systems is proved in [10]. In [4], the optimality conditions are analysed. The only restriction 
of this filter is to impose that the dimension of the outputs is greater than the inputs $(n>L)$, to ensure the uniqueness of the estimated variables and for the extended case $n$ should be greater or equal to $m+L$ where $m$ is the length of the parameters vector. Unfortunately, like the KF, the limitations of this filter are the hypotheses on the model and measurements noises and the need for a perfect knowledge of covariance matrices $W$, and $V$. If the latter can be known for some applications, $W$ can never be. In this work, $W$ is approximated using a recursive least-squares approach [11]:

$$
W(k)=\left(\lambda^{-1}-1\right) P_{x}(k \mid k) .
$$

where $\lambda$ is the forgetting factor, setting how much old measurements can be neglected, and preventing the Kalman gain from converging to zero.

\section{B. Simulation and experimental results}

Simulations with computed and experimental data are carried out to evaluate the reconstruction performance of the adopted method. In both cases, the dissipation parameter $\tau$ is assumed to be known and constant, given by [12] empirical model. This assumption leads to a low-order of the problem conditioning number.

1) Illustrative example:: The simulated data is generated using:

$\left\{\begin{array}{l}\chi_{e}(z, t)=\left(0.1+5 z+2 z^{2}+4 z^{3}\right) \mathbf{1}(t)\left(m^{2} / s\right) ; \tau=0.05(s) \\ S(z, t)=\frac{10^{5}}{\sqrt{2 \pi \sigma}} \exp \left(\frac{-(z-\mu)^{2}}{2 \sigma^{2}}\right) \mathbf{1}(t)(M W) \\ z \in[0,1], \quad t \in[0,1], \quad d z=0.05, \quad d t=0.01 .\end{array}\right.$

where the operator $\mathbf{1}(t)$ indicates that $\chi_{e}$ and $S$ are constant in time. The choice of $\chi_{e}, \tau$ and $S$ is motivated by the example proposed by [13]. It was assumed that the diffusion coefficient has a monomial monotonically increasing function and the heating source undergoes a spatial Gaussian form. These parameters were considered constant in time. Fig. 2 shows that using a Legendre basis of minimum admissible dimension $m=4$, the approximation error of $\chi_{e}$ is of the order of $10^{-15}$, and using a cubic B-splines basis of order $L=10$, the relative approximation error of $S$ is around $10^{-4}$. This led us to take $n \geq 14$ for the temperature basis.
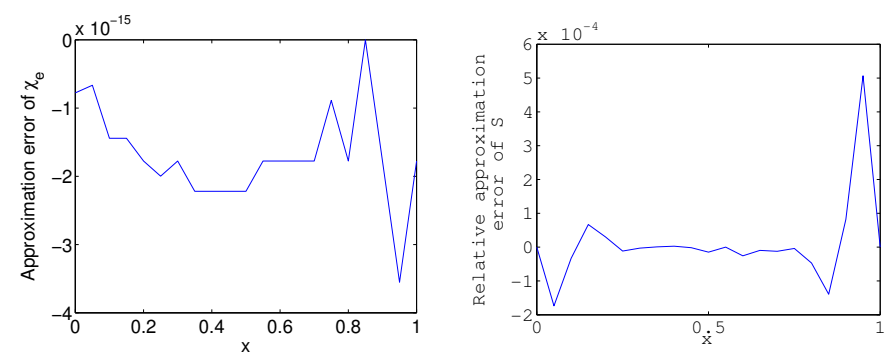

Fig. 2: Approximation errors of $\chi_{e}$ and $S$ for the simulated example
To evaluate the reconstruction performance using the EKFUI-WDF, the initialization of the filter was arbitrary. The covariance matrix of the model noise $W$ is given by (15) with $\lambda=0.9$, the measurements covariance noise $V$ is set to $10^{-3} I_{n}$ and the state error covariance matrix is $10 I_{n+M}$.
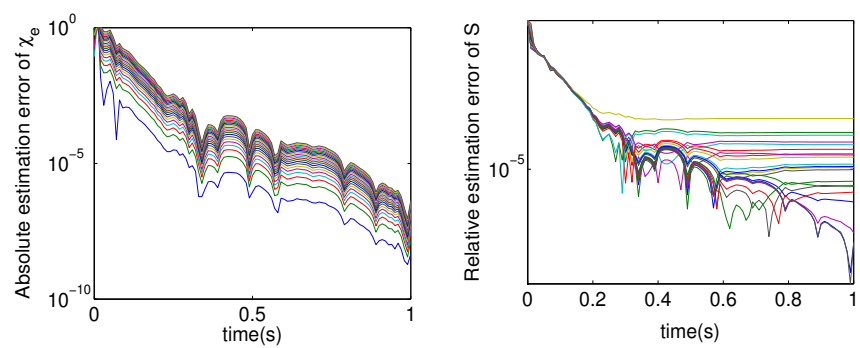

Fig. 3: Estimation errors of $\chi_{e}$ and $S$ for the simulated example
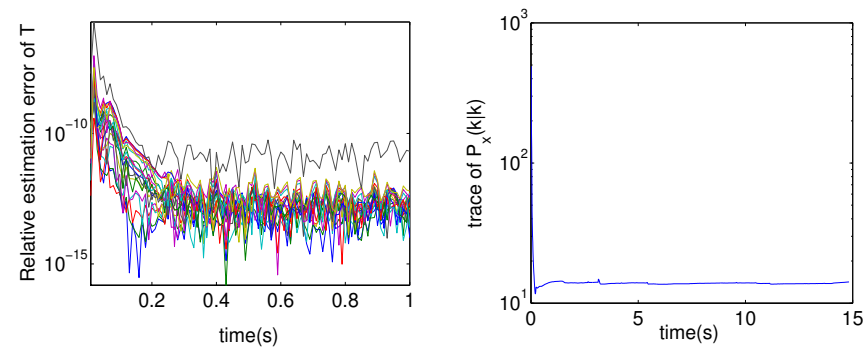

Fig. 4: Relative estimation error of the temperature profile $T$ and the trace of $P_{x}(k \mid k)$ for the simulated example

From Fig. 3 and Fig. 4, the estimation of $\chi_{e}, S$ and $T$ using the EKF-UI-WDF is very good. The filter needs only few iterations to converge exponentially to the original variables, the trace of the estimation error covariance matrix is an exponential decreasing function of time and converges to $10^{-3}$.

2) Experimental results: Tore Supra is a large tokamak with a superconducting toroidal magnet with plasma minor radius $a=0.72 \mathrm{~m}$ and major radius $R=2.4 \mathrm{~m}$. We consider the discharge TS 33632, where heating is mainly due to the radio-frequency power at the Ion Cyclotron Resonant Heating (ICRH) frequency. For the projection method, like in Section II-B, the temperature projection basis is $n=20$. For $\chi_{e}$ and $S$, the bases orders are $m=L=10$. Simulations show that even if larger bases dimensions are considered the improvement is negligible while the conditioning number is increased. Fig. 5 presents the estimated profiles of $\chi_{e}$ and $S$ in the spatial validity interval $(z \leq 0.8)$. Both are positive without enforcing this constraint in the Kalman filter criterion. The EKF-UI-WDF performs well since the relative estimation error of $T$ in Fig. 6 converges to $10^{-3}$ on average, the trace of $P_{x}(k \mid k)$ is decreasing and the standard deviation converges to $0.5 \mathrm{eV}$. Integrating the estimated source term $S$ over the plasma volume using (17) gives the estimated 

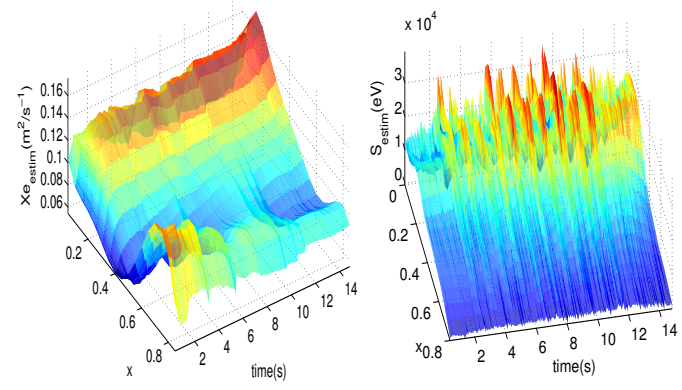

Fig. 5: Estimated profiles of $\chi_{e}$ and $S$
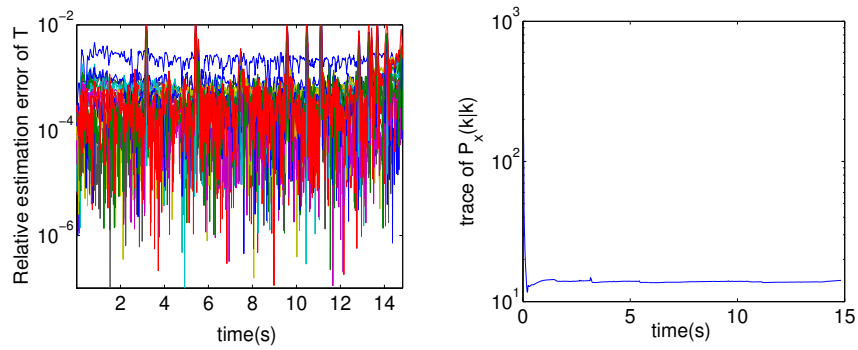

Fig. 6: Relative estimation error of $T$ and the trace of $P_{x}(k \mid k)$

absorbed power denoted by Estimated $P_{e}$.

$$
\text { Estimated } P_{e}=6 e \pi^{2} a^{2} R \int_{0}^{1} n(x, t) S(x, t) x d x,
$$

where $e$ is the electron's charge and $n$ is the measured electron density. The superposition of the input power ( $P_{e}$, in red dashed-line) and the absorbed estimated power (Estimated $P_{e}$, continuous blue line) is presented in Fig.7. The temporal power form is consistent with the estimated, with a different magnitude probably due to energy losses (equipartition with ions, convection, radiation).

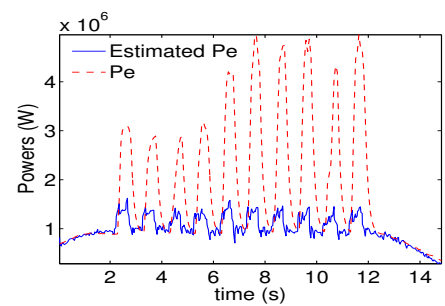

Fig. 7: $P_{e}$ and Estimated $P_{e}$

\section{CONCLUSION}

In this paper we discussed a method of estimating distributed parameters in a finite dimensional framework for linear parabolic PDEs. First we dealt with the direct problem, which consists in proving its well posedness and then developing an approximate solution using the spectral-Galerkin method. Under the Ritz-Galerkin formulation, the spatiotemporal problem was reduced to a state-space time-varying parameter model, and then the EKF-UI-WDF was used to estimate simultaneously the states, the parameters and the inputs. The major difficulty is the choice of the projection basis: a trade-off between precision and stability of the solution has to be done. Simulation and experimental results testified the interest of the adopted methodology. To overtake the EKF restrictions, other filters like the UKF can be used combined with the square-root implementation to guarantee the stability and to fix the conditioning number problem. This strategy will be the subject of future works.

\section{ACKNOWLEDGEMENT}

This work was carried out within the framework of the European Fusion Development Agreement and the French Research Federation for Fusion Studies. It is supported by the European Communities under the contract of Association between EURATOM and CEA. The views and opinions expressed herein do not necessarily reflect those of the European Commission.

\section{REFERENCES}

[1] P. K. Kitanidis, "Unbiased minimum-variance linear state estimation," Automatica, vol. 23, no. 6, pp. 775-778, 1987.

[2] M. Darouach, M. Zasadzinskind, and M. Boutayeb, "Extension of minimum-variance estimation for systems with unknown inputs," Automatica, vol. 39, pp. 867-876, 2003.

[3] S. Gillijns and B. D. Moor, "Unbiased minimum-variance estimation for systems with unknown inputs," Automatica, vol. 43, pp. 934-937, 2007.

[4] S. Pan, H. Su, H. Wang, J. Chu, and R. Lu, "Input and state estimation for linear systems: A least squares estimation approach," in Proceedings of the 7th Asian Control Conference, Hong Kong, China, August 27-29 2009, pp. 378-383.

[5] F. Hinton and R. Hazeltine, "Theory of plasma transport in toroidal confinement systems," Reviews of Modern Physics, vol. 48, no. 2, pp. 239-308, 1976

[6] A. Iserles and S. Nørsett, "From high oscillation to rapid approximation i: Modified Fourier expansions," IMA Journal of Numerical analysis, vol. 28, pp. 862-887, 2006.

[7] H. Banks, P. Kareiva, and P. Lamm, "Modeling insect dispersal and estimating parameters when mark-release techniques may cause initial disturbances," Journal of Mathematical Biology, vol. 22, pp. 259-277, 1985.

[8] W. Yu and J. H. Seinfeld, "Identification of parabolic distributed parameter systems by regularization with differential operators," Journal of mathematical analysis and applications, 1988.

[9] A. A Oberai, N. H. Gokhale, and G. R. Feijóo, "Solution of inverse problems in elasticity imaging using the adjoint method," Inverse Problems, vol. 19, pp. 297-313, 2003.

[10] L. Ljung, "Asymptotic behavior of extended Kalman filter as a parameter estimator for linear systems," IEEE Trans. on Auto. Control, vol. AC-24, no. 1, pp. 36-50, 1979.

[11] E. A. Wan and A. T. Nelson, Random Analysis and Kalman Filtering. John Wiley \& Sons, Inc., 2001, ch. Dual Extended Kalman Filter Methods, pp. 123-175.

[12] G. Hoang, B. Saoutic, L. Guiziou, V. Basiuk, A. Bécoulet, R. Budny, F. Clairet, L. Colas, P. Devynck, C. Gil, E. Joffrin, X. Litaudon, J.L. Ségui, I. Voitsekhovitch, and X. Zou, "Magnetic shear effects on confinement and electron heat transport in Tore Supra discharges with electron heating," Nuclear Fusion, vol. 38, no. 1, pp. 117-132, 1998.

[13] X. L. Zou, G. Giruzzi, J. F. Artaud, F. Bouquey, A. Clémonçon, C. Darbos, R. J. Dumont, C. Guivarch, M. Lennholm, R. Magne, and J. L. Ségui, "Electron heat transport and ECRH modulation experiments in Tore Supra tokamak," Nuclear Fusion, vol. 43, pp. 1411-1420, 2003. 